Supplement of Arch. Anim. Breed., 62, 9-32, 2019 https://doi.org/10.5194/aab-62-9-2019-supplement (C) Author(s) 2019. This work is distributed under the Creative Commons Attribution 4.0 License.

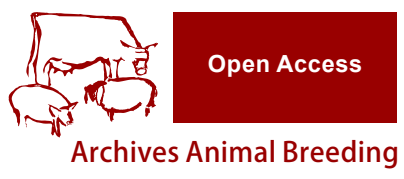

(c) (1)

Supplement of

\title{
Comprehensive assessment of candidate genes associated with fattening performance in Holstein-Friesian bulls
}

Sena Ardicli et al.

Correspondence to: Faruk Balci (fbalci@uludag.edu.tr)

The copyright of individual parts of the supplement might differ from the CC BY 4.0 License. 
Table S1 Detailed information about the studied genes and single nucleotide polymorphisms (SNPs)

\begin{tabular}{|c|c|c|c|c|c|c|c|c|}
\hline Gene & BTA & Localization & $\begin{array}{c}\text { NCBI Gene } \\
\text { ID }\end{array}$ & $\begin{array}{c}\text { Genbank } \\
\text { Accession } \\
\text { Number } \\
\end{array}$ & SNP Name* & SNP Localization & Allele & $\begin{array}{l}\text { Amino Acid } \\
\text { Alterations }\end{array}$ \\
\hline \multirow[t]{2}{*}{$L E P$} & \multirow{2}{*}{4} & \multirow{2}{*}{$92,436,922-92,453,653$} & \multirow{2}{*}{280836} & \multirow[t]{2}{*}{ AF536174.1 } & A80V & Exon 3 & $\mathrm{C} / \mathrm{T}$ & Ala/Val \\
\hline & & & & & g. $3691 \mathrm{G}>\mathrm{A}$ & Exon 3 & $\mathrm{G} / \mathrm{A}$ & Val/Met \\
\hline \multirow[t]{2}{*}{$F A B P 4$} & \multirow[t]{2}{*}{14} & \multirow{2}{*}{$44,676,542-44,681,059$} & \multirow[t]{2}{*}{281759} & \multirow[t]{2}{*}{ NC_007312.4 } & g. $2834 \mathrm{C}>\mathrm{G}$ & Intron 1 & $\mathrm{C} / \mathrm{G}$ & - \\
\hline & & & & & g. $3533 \mathrm{~T}>\mathrm{A}$ & Intron 2 & $\mathrm{~T} / \mathrm{A}$ & - \\
\hline$D G A T 1$ & 14 & $603,813-612,791$ & 282609 & AY065621 & $\mathrm{K} 232 \mathrm{~A}^{* *}$ & Exon 8 & $\mathrm{~K} / \mathrm{A}$ & Lys/Ala \\
\hline$T G$ & 14 & $8,217,490-8,453,614$ & 280706 & X05380 & $\mathrm{C} 422 \mathrm{~T}$ & 5'UTR & $\mathrm{C} / \mathrm{T}$ & - \\
\hline$I G F 1$ & 5 & $66,191,602-66,264,083$ & 281239 & AF210383 & $\mathrm{C} 472 \mathrm{~T}$ & 5'UTR & $\mathrm{C} / \mathrm{T}$ & - \\
\hline$I G F 1 R$ & 21 & 7,780,293-8,080,394 & 281848 & U33122 & G404T & Intron 12 & $\mathrm{~A} / \mathrm{B}$ & - \\
\hline MYF5 & $5 q 13$ & $10,284,434-10,287,669$ & 281335 & M95684 & g. $1911 \mathrm{~A}>\mathrm{G}$ & Intron 2 & $\mathrm{~A} / \mathrm{G}$ & - \\
\hline$L G B^{* * *}$ & $11 \mathrm{q} 28$ & $103,255,824-103,264,276$ & 280838 & AC_000168.1 & T5261C & Exon 4 / Intron 4 & $\mathrm{~A} / \mathrm{B}$ & Asp/Val; Gly/Ala \\
\hline \multirow{2}{*}{ CAPN1 } & \multirow{2}{*}{29} & \multirow{2}{*}{$43,400,333-43,427,397$} & \multirow{2}{*}{281661} & \multirow{2}{*}{ AF252504 } & G316A & Exon 9 & $\mathrm{C} / \mathrm{G}$ & Gly/Ala \\
\hline & & & & & V530I & Exon 14 & $\mathrm{~A} / \mathrm{G}$ & Val/Ile \\
\hline$C A S T$ & 7 & $96,033,978-96,167,151$ & 281039 & AF117813 & $\mathrm{S} 20 \mathrm{~T}$ & Exon $1 \mathrm{C} / 1 \mathrm{D}$ & $\mathrm{G} / \mathrm{C}$ & Ser/Thr \\
\hline$G H R$ & $20 \mathrm{q} 17$ & $31,868,624-32,178,311$ & 280805 & AF140284 & S555G & Exon 10 & $\mathrm{G} / \mathrm{A}$ & Ser/Gly \\
\hline OLRI & 5 & $99,803,497-99,815,138$ & 281368 & NM_174132 & g. $8232 \mathrm{C}>\mathrm{A}$ & 3'UTR & $\mathrm{A} / \mathrm{C}$ & - \\
\hline
\end{tabular}

$L E P$ - leptin. FABP4 - fatty acid binding protein 4. DGATI - diacylglycerol acyltransferase 1. TG - thyroglobulin. IGF1 - insulin-like growth factor $1 . I G F 1 R$ - insulin-like growth factor receptor 1 . MYF5 - myogenic factor 5. LGB - beta-lactoglobulin. CAPN1 - micromolar calcium-activated neutral protease 1 . CAST - calpastatin. GHR growth hormone receptor. $O L R I$ - oxidized low-density lipoprotein receptor.

*Marker names were selected according to genomic regions or amino acid alterations according to HGSV.

**The two SNP in the DGAT1 gene lie immediately adjacent to one another in exon 8, and the two alleles at this locus are AA and GC, encoding lysine (K) and alanine (A) at the amino acid position 232.

***Also known as progestagen-associated endometrial protein $(P A E P)$. 
Table S2 Estimates of variance components of the markers showing significant associations

\begin{tabular}{|c|c|c|c|c|c|}
\hline Trait & $\begin{array}{l}\text { Corresponding } \\
\text { Markers }\end{array}$ & $\begin{array}{l}\text { Additive } \\
\text { Effect }^{1,2}\end{array}$ & $\begin{array}{l}\text { Dominant } \\
\text { Effect }^{1,3}\end{array}$ & $\begin{array}{c}\text { Presence of } \\
\text { Overdominance }^{1,4}\end{array}$ & $\begin{array}{c}\text { Overall } \\
P \text { value } \\
1,5\end{array}$ \\
\hline DTRW2 & $L E P$ & $-8.67 * * *$ & -2.43 & & 0.001 \\
\hline \multirow[t]{3}{*}{ DTRW3 } & $L E P$ & -7.32 & $8.09 * *$ & & 0.008 \\
\hline & $F A B P 43691$ & -18.44 & $-16.76^{*}$ & & 0.037 \\
\hline & $G H R$ & 6.75 & $6.31 *$ & & 0.034 \\
\hline DTRW4 & CAPN1 316 & -13.36 & $-33.04 * *$ & + & 0.005 \\
\hline DTRW5 & $L E P$ & $-10.91 * *$ & 5.77 & & 0.006 \\
\hline \multirow{3}{*}{ W2-W3 FP } & FABP4 2834 & $-4.37 *$ & -0.02 & & 0.015 \\
\hline & $L G B$ & 3.45 & $2.47 *$ & & 0.047 \\
\hline & $G H R$ & 2.88 & $3.52 * *$ & & 0.006 \\
\hline W4-W5 FP & $G H R$ & -3.45 & $9.25^{*}$ & + & 0.019 \\
\hline FW & CAPN1 316 & 21.66 & $18.13 * *$ & & 0.003 \\
\hline \multirow[t]{2}{*}{ W2-W3 DMI } & $C A S T$ & -13.89 & $16.67 *$ & & 0.042 \\
\hline & $G H R$ & 17.23 & $15.66 * *$ & & 0.006 \\
\hline \multirow[t]{3}{*}{ TDMI } & $L E P$ & -26.01 & $159.00 *$ & & 0.018 \\
\hline & $I G F I$ & 55.50 & $-94.51 *$ & + & 0.020 \\
\hline & $G H R$ & $155.20 * * *$ & -40.50 & & 0.000 \\
\hline W1-W2 DDMI & $F A B P 43533$ & 0.13 & $-0.12 * *$ & & 0.004 \\
\hline \multirow[t]{2}{*}{ W4-W5 DDMI } & MYF5 & 0.01 & $0.39 *$ & + & 0.034 \\
\hline & $G H R$ & 0.16 & $-0.48 *$ & + & 0.040 \\
\hline \multirow[t]{4}{*}{ TDDMI } & $L E P$ & $-0.71 * * *$ & -0.12 & & 0.000 \\
\hline & $F A B P 43533$ & -0.14 & $-0.28 *$ & + & 0.011 \\
\hline & $I G F 1$ & 0.14 & $-0.19 *$ & + & 0.020 \\
\hline & $L G B$ & -0.19 & $-0.26^{*}$ & + & 0.012 \\
\hline \multirow{2}{*}{ W2-W3 FCR } & CAST & -0.16 & $0.18^{*}$ & & 0.023 \\
\hline & $G H R$ & 0.21 & $0.22 * *$ & & 0.004 \\
\hline W3-W4 FCR & $O L R 1^{6}$ & - & - & & 0.024 \\
\hline W4-W5 FCR & FABP4 3533 & -0.06 & $-0.77 *$ & + & 0.031 \\
\hline \multirow[t]{3}{*}{ TFCR } & $L E P$ & -0.18 & $0.19 *$ & & 0.048 \\
\hline & $I G F 1$ & -0.42 & $0.30 * *$ & & 0.004 \\
\hline & CAPN1 316 & -0.62 & $-0.56 * * *$ & & 0.001 \\
\hline \multirow[t]{3}{*}{ W2-W3 ADWG } & $F A B P 42834$ & $0.05^{*}$ & -0.01 & & 0.019 \\
\hline & $L G B$ & $-0.04 *$ & -0.02 & & 0.036 \\
\hline & $G H R$ & -0.04 & $-0.03 * *$ & & 0.009 \\
\hline \multirow[t]{2}{*}{ W4-W5 ADWG } & $F A B P 43533$ & 0.03 & $0.13 *$ & + & 0.019 \\
\hline & $G H R$ & 0.06 & $-0.13^{*}$ & & 0.047 \\
\hline TADWG & CAPN1 316 & 0.05 & $0.04 * *$ & & 0.007 \\
\hline
\end{tabular}

$\mathrm{W} 1$ - $100 \mathrm{~kg}, \mathrm{~W} 2-200 \mathrm{~kg}, \mathrm{~W} 3-300 \mathrm{~kg}, \mathrm{~W} 4-400 \mathrm{~kg}$, W5 - $450 \mathrm{~kg}$ target body weight. DTR - days to reach. FP - fattening period. FW - final weight. DMI - dry matter intake. TDMI - total dry matter intake throughout the entire experimental period. DDMI - daily dry matter intake. TDDMI - total daily dry matter intake throughout the entire experimental period. FCR - feed conversion rate. TFCR - total feed conversion rate throughout the entire experimental period. ADWG - average daily weight gain. TADG - total average daily weight gain throughout the entire experimental period.

${ }^{1}$ Least squares means for each genotype are shown in Tables 5-7.

${ }^{2}$ Additive effect is estimated as the difference between the 2 homozygous means divided by 2 (a) (Falconer and Mackay, 1996).

${ }^{3}$ Dominance effect is estimated as the nonadditive genetic effects or the deviation of the heterozygote from the mean of the 2 homozygotes (d) (Falconer and Mackay, 1996).

${ }^{4}$ The degree of dominance may be expressed as $\mathrm{d} / \mathrm{a}$. If there is over dominance, $\mathrm{d}$ is greater than $+\mathrm{a}$ or less than $-\mathrm{a}$ (Falconer and Mackay, 1996).

${ }^{5}$ Overall $P$ value for marker as a fixed genotype effect.

${ }^{6}$ Only two genotypes were observed for $O L R 1$ marker, accordingly, additive and dominance effect could not be estimated.

$* P<0.05, * * P<0.01, * * * P<0.001$ 